\title{
Efeitos de Instrução e de Treino Parental em Cuidadores de Crianças com Câncer ${ }^{1}$
}

\author{
Inaê Benchaya ${ }^{2}$ \\ Eleonora Arnaud Pereira Ferreira \\ Izabel Cristina da Silva Brasiliense \\ Universidade Federal do Pará
}

\begin{abstract}
RESUMO - Esta pesquisa teve como objetivo analisar os efeitos de instrução e de treino parental sobre comportamentos observados em cuidadores e em crianças com diagnóstico de câncer durante procedimento de punção venosa em ambulatório. Participaram nove cuidadores em três condições (Rotina, Manual e Treino). Fez-se análise de características familiares, estilo parental, efeitos de um manual de instruções e de treino parental, com sessões de observação direta do comportamento durante punção venosa. Os resultados apontam efeito positivo do manual para mudança de comportamento em curto prazo. Após treino parental, observou-se aumento nas taxas de monitoria positiva do cuidador, com relatos de generalização desses comportamentos para outros contextos. Discute-se a importância do estilo parental como fator de proteção à criança com câncer.
\end{abstract}

Palavras-chave: distresse comportamental, instrução, treino parental, câncer infantil

\section{Effects of Instruction and Parental Training for Caregivers of Children with Cancer}

\begin{abstract}
This research aimed to examine the effects of parental training and instruction on observed behaviors in caregivers and children diagnosed with cancer during the venipuncture procedure on an outpatient basis. Nine caretakers participated in three different conditions (Routine, Manual and Training). Family characteristics, parental style, and effects of manual and parental training were analyzed with direct observation of behavior during the venipuncture procedure of children. After parental training, the results showed an increase in the positive effect of the manual for short term behavior change, and an increase in the positive monitoring rates of the caregiver, with reports of generalizations of these behaviors for other contexts. The importance of parenting style as a protective factor for children with cancer was discussed.
\end{abstract}

Keywords: behavior distress, instruction, parent coaching, childhood cancer

É frequente encontrar na literatura comentários de que muitas crianças em tratamento para o câncer não se habituam aos procedimentos invasivos associados ao tratamento, mesmo depois de repetidas exposições (Costa Junior, 1999). Esse fato sugere a necessidade de se realizar intervenções psicológicas de manejo da dor, de ensino de estratégias de enfrentamento através de exercícios e/ou uso de informações a fim de que os pacientes infantis e seus cuidadores aumentem a frequência de comportamentos de colaboração e reduzam a ocorrência de distresse comportamental (Lemos \& Ferreira, 2010). O termo distresse comportamental tem sido utilizado para caracterizar um conjunto de reações comportamentais de desconforto (ou sofrimento) frente a procedimentos médicos invasivos, tais como respostas indicadoras de dor, ansiedade e medo (Jay, Ozolins, \& Elliot, 1983).

Uma das estratégias de enfrentamento adotadas com crianças que já dispõem de repertório verbal é o oferecimento de informações acerca da doença e do tratamento, por meio de materiais educativos (e.g. manuais e folhetos). Para Torres, Candido, Alexandre e Pereira (2009), quando utilizados de forma eficiente - isto é, com instruções básicas

1 Apoio: CAPES.

2 Endereço para correspondência: Travessa 9 de Janeiro, 199, Apt. 502, Umarizal, Belém - PA. CEP 66060-370.E-mail: inacbd@gmail.com. e esclarecedoras - esses materiais permitem ao paciente e ao seu cuidador um guia de orientações para esclarecimento de dúvidas, tomada de decisão sobre procedimentos, maximização do tempo e da qualidade das consultas com profissionais de saúde. Para Ferreira (2005), também podem favorecer mudança de comportamentos de adesão ao tratamento, por meio da instalação de repertório de autocuidado e de discriminação de sintomas.

Aliadas ao uso de informações, técnicas cognitivas e comportamentais têm sido apontadas como contingências que podem alterar a função de estímulos presentes no ambiente de tratamento. Tais técnicas podem exercer controle sobre os comportamentos da criança durante a execução de procedimentos invasivos (Chen, Joseph, \& Zeltzer, 2000).

Em função dos complexos fatores envolvidos no manejo de comportamentos de crianças expostas a procedimentos invasivos, muitos estudos passaram a focalizar técnicas de distração, relaxamento/respiração, modelação, reforçamento por contingência, ensaio comportamental, além do uso de informações (Linhares \& Doca, 2010). No entanto, alguns aspectos devem ser considerados para a escolha e uso das técnicas cognitivas e comportamentais. A história prévia, a idade e o repertório comportamental de enfrentamento da criança também devem ser considerados (Christensen \& Fatchet, 2002). Além da criança, profissionais de saúde e, 
especialmente, pais/cuidadores podem ser treinados para auxiliar no enfrentamento de procedimentos invasivos (Vance \& Eiser, 2004).

Rodriguez et al. (2012) observaram correlações positivas entre o distresse dos pais e aquele manifestado pela criança com câncer. Por sua vez, Herman e Miyazaki (2007) apontam a importância de se desenvolver intervenções comportamentais focalizadas diretamente sobre os pais (parent coaching), como uma forma de se alcançar resultados mais eficazes no que diz respeito à ampliação do repertório de enfrentamento da criança durante procedimento invasivo. Sugerem ainda a inclusão do treino de outras habilidades (como demonstração de cuidado e carinho), garantindo melhor qualidade na relação com a criança assim como generalização para outros ambientes.

Algumas vantagens do treino dos pais/cuidadores podem ser destacadas, para o uso de distração com a criança: 1) estarem livres para tentar atrair a atenção da criança, já que não possuem a responsabilidade de conduzir o procedimento invasivo; 2) conhecerem os estímulos que podem atrair a atenção da criança; e, 3) uma vez que aprendam a empregar a técnica da distração, os pais podem generalizar essas habilidades para outros contextos (Christensen \& Fatchet, 2002).

É importante destacar que a influência dos pais não se restringe apenas ao contexto hospitalar, mas a outros ambientes em que a criança possa interagir. Práticas educativas utilizadas pelos pais no controle e orientação dos comportamentos dos filhos podem influenciar a forma como a criança irá se comportar frente a situações estressoras (Piccinini, Castro, Alvarenga, Vargas, \& Oliveira, 2003).

Os estilos parentais têm sido tradicionalmente classificados pela psicologia do desenvolvimento em três amplos grupos: autoritário, permissivo e autoritativo. O estilo autoritário é caracterizado por alta frequência de comportamentos de controle sobre os comportamentos dos filhos, baixa frequência de comportamentos de demonstração de afeto e diálogo, e uso de medidas punitivas (Weber, Prado, Viezzer, \& Brandenburg, 2004). Pais autoritários demonstram maior ansiedade frente a procedimentos invasivos, especialmente quando as crianças apresentam altos níveis de distresse, não demonstrando habilidades de enfrentamento ou repertório afetivo (carinho) que auxiliem a diminuir o medo ou a ansiedade da criança (Peterson et al., 2007).

$\mathrm{O}$ estilo permissivo (subdividido em indulgente e permissivo) é o mais encontrado entre cuidadores de crianças enfermas (Piccinini et al., 2003). Nesse estilo, os pais não utilizam medidas punitivas, mas também não possuem comportamentos de direcionamento, nem modelação em relação ao comportamento dos filhos. Assim, tendem a apresentar maior número de atrasos e cancelamento às consultas, baixa adesão aos cuidados, prejudicando não só o tratamento em si, mas não possibilitando a aprendizagem de comportamentos de autocuidado e responsabilidade da criança pela sua saúde (Vance \& Eiser, 2004; Wakefield et al., 2010).

No estilo autoritativo, os cuidadores educam de maneira racional e orientada, com alta frequência de carinho e cuidado, assim como diálogo com os filhos. Esses pais possibilitam tanto o adequado desenvolvimento físico e psicológico da criança (Sapienza, Aznar-Farias, \& Silvares, 2009), quanto disponibilizam os cuidados necessários durante o tratamento de doenças crônicas, favorecendo a pontualidade em consultas, adesão ao tratamento e comportamentos de suporte emocional e enfrentamento durante procedimentos invasivos (Vance \& Eiser, 2004; Wakefield et al., 2010).

A classificação ampla dos estilos parentais inclui um conjunto de práticas específicas que, de acordo com sua frequência, podem caracterizar um ou outro estilo. Gomide (2006) divide as práticas educativas parentais em dois grupos. As práticas que contribuem para o desenvolvimento do comportamento pró-social - denominadas práticas positivas - são a monitoria positiva e o comportamento moral. E as práticas educativas negativas, que quando usadas frequentemente tendem a desenvolver comportamento antissocial, são: a monitoria negativa, a negligência, a disciplina relaxada, o abuso físico e a punição inconsistente.

Assim, observa-se que diante da doença e da percepção do sofrimento da criança com câncer, os pais podem apresentar dificuldades na forma de lidar com o filho. A literatura mostra que os pais tendem a desenvolver sentimentos "de pena e culpa" que os levam a adotar comportamentos excessivamente permissivos, deixando de impor limites e facilitando o desenvolvimento de problemas de controle de comportamento (Herman \& Miyazaki, 2007).

Nesse sentido, o estilo parental adotado pelos pais e/ou cuidadores é considerado importante para o desenvolvimento de comportamentos saudáveis da criança, especialmente as portadoras de doenças crônicas, como o câncer. Assim, práticas parentais adequadas podem contribuir para auxiliar o manejo diário de uma criança que, além de apresentar necessidades próprias do desenvolvimento, também necessita atender a demanda de cuidados especiais provenientes de seu estado de saúde alterado (Herman \& Miyazaki, 2007).

Embora estudos sobre o tema sejam recorrentes, não está claro quais os recursos mais eficazes para promover um ambiente de cuidados à criança com câncer, em especial durante a realização de procedimentos dolorosos ligados ao tratamento, como é o caso da punção venosa para quimioterapia. Este estudo investigou os efeitos da rotina, do uso de instruções e de treino parental sobre comportamentos observados em cuidadores e em crianças com câncer durante a realização de punção venosa para quimioterapia em ambulatório.

\section{Método}

\section{Participantes}

Foram selecionados nove cuidadores de crianças com câncer em tratamento quimioterápico no ambulatório de um hospital especializado no tratamento de doenças crônico-degenerativas. A seleção ocorreu por meio de convites orais aos acompanhantes e crianças presentes no ambulatório. Foram incluídos na pesquisa aqueles que declararam ser o cuidador principal da criança e assinaram o Termo de Consentimento Livre e Esclarecido (TCLE). Foram excluídos aqueles com dificuldade para compreender o procedimento do estudo e os cuidadores de crianças que não foram submetidas exclusivamente a quimioterapia. 
Foram feitos 60 convites, durante sete meses, dos quais cinco acompanhantes recusaram participar da pesquisa, 20 foram excluídos a partir dos critérios utilizados, 35 cuidadores aceitaram participar dos quais 26 foram excluídos por dificuldades em completar a coleta de dados devido a intercorrências no tratamento da criança (e.g. uso de cateter, internações, óbito).

Todos os participantes eram pais biológicos das crianças, sendo sete mães e dois pais, com idades entre 22 e 43 anos $(\mathrm{M}=30$ anos, $\mathrm{DP}=7,8)$. De acordo com a classificação da Associação Brasileira de Empresas de Pesquisa [ABEP] (www.abep.org), a maioria se encontrava na classe econômica $C(n=6)$, com dois na classe D e um na classe B. Quanto à escolaridade, a maioria tinha Ensino Fundamental incompleto $(n=5)$, seguido por Ensino Médio completo $(n=2)$, e apenas um participante informou ter concluído uma pós-graduação (mestrado). Considerando a ocupação, somente dois exerciam atividade remunerada, os demais $(n=7)$ haviam se afastado do emprego ou de suas atividades por conta do tratamento da criança.

Quanto às crianças, a amostra foi composta por duas meninas e um menino em cada uma das três condições, com idades entre 4 a 9 anos $(M=5,8$ anos; $\mathrm{DP}=1,5)$, sendo quatro pré-escolares e cinco escolares. Em relação ao diagnóstico, a maioria tinha Leucemia Linfoblástica Aguda [LLA] $(n=4)$, seguida de Linfoma $(n=3)$, e de Nefroblastoma $(\mathrm{n}=2)$. Independentemente do diagnóstico, todas as crianças realizavam quimioterapia em ambulatório e haviam iniciado o tratamento por um período entre um e cinco meses $(\mathrm{M}=3$ meses, $\mathrm{DP}=1,5)$.

\section{Instrumentos e materiais}

a) Roteiro de entrevista. Utilizado para coletar informações sobre histórico de tratamento e hospitalizações da criança; reações frente a procedimentos médicos invasivos, características familiares, assim como condição socioeconômica.

b) Instrumento de Avaliação das Relações Familiares (Parental Bonding Instrument [PBI]). Elaborado por Parker, Tupling e Brown (1979), foi adaptado para o português brasileiro por Herman e Miyazaki (2007). Neste estudo utilizou-se a versão descrita por Rezende (2001), na qual o informante avalia suas próprias práticas parentais. O instrumento classifica os estilos parentais em autoritativo, autoritário, indulgente e negligente, de acordo com quatro dimensões (dependência, autonomia, rejeição e negligência).

c) Escala de Avaliação Comportamental denominada originalmente como Observation Scale of Behavior Distress (OSBD). Elaborada por Jay et al. (1983), foi adaptada no Brasil por Borges (1999) e Costa Junior (2001). Contêm categorias morfológicas de comportamento, definidas operacionalmente, que abrangem respostas verbais, vocais e motoras da criança, indicativas do sofrimento experienciado em situações de procedimentos médicos invasivos, assim como as do seu cuidador. $\mathrm{O}$ registro das categorias contidas na OSBD é feito a cada intervalo de 15 segundos, em um sistema de checklist, em que cada categoria é registrada como presente ou ausente. Para este estudo, as categorias foram adaptadas de acordo com os dados obtidos, tendo como base as categorias prévias criadas por Costa Junior (2001).

d) Manual de orientação para cuidadores sobre punção venosa em crianças. Elaborado por Brasiliense (2012), tem o objetivo de instruir cuidadores de crianças em tratamento quimioterápico com informações acerca do que é quimioterapia e punção venosa, com instruções e sugestões (através de figuras) de dicas de comportamentos para os cuidadores emitirem no contexto do procedimento.

e) Protocolo para treino parental. Elaborado para este estudo com base em Pinheiro, Del Prette e Haase (2002); consta de três encontros programados para auxiliar os pais a desenvolverem habilidades sociais com vistas a desempenharem papel de coterapeutas no momento da punção venosa, auxiliando a criança no enfrentamento do procedimento invasivo, e também promovendo melhor relacionamento entre o cuidador e a criança (para descrição mais detalhada consultar Duarte, 2012).

\section{Procedimento}

Após a aprovação do projeto pelo hospital e pelo Comitê de Ética em Pesquisa, iniciou-se a fase piloto para treino das pesquisadoras nos instrumentos de coleta de dados e para ambientação ao contexto de observação. Em seguida, foram feitos convites aos cuidadores de crianças presentes no ambulatório de quimioterapia do hospital para a participação na pesquisa.

Com a obtenção do consentimento dos cuidadores, fez-se a primeira observação de uma sessão de punção venosa para a realização de quimioterapia na qual os comportamentos da criança e do cuidador foram considerados como linha de base. A seguir, aplicou-se o Roteiro de Entrevista e o PBI com o cuidador. Após a aplicação desses instrumentos, os participantes foram distribuídos, por ordem de ingresso no estudo, em uma de três condições.

\section{Condição Rotina}

Nessa condição, três participantes (P1, P2 e P3) foram submetidos a três sessões de observação direta realizadas em ambulatório durante procedimento de punção venosa para quimioterapia com a criança, com intervalo médio de duas semanas entre as sessões.

\section{Condição Manual}

Três participantes (P4, P5 e P6) receberam o Manual de orientações sobre quimioterapia e punção venosa, o qual foi lido individualmente e em conjunto com a pesquisadora de modo a esclarecer quaisquer dúvidas. Após duas semanas, foram realizadas três sessões de observação direta do proce- 
dimento de punção venosa para quimioterapia com a criança, em intervalo de, no mínimo, uma semana entre elas.

\section{Condição Treino}

Três participantes (P7, P8 e P9) também receberam e leram, individualmente e junto com a pesquisadora, o mesmo Manual. Posteriormente, realizou-se a segunda sessão de observação direta da punção venosa com a criança. A partir dos resultados obtidos por meio do PBI, os cuidadores dessa condição foram classificados em estilo parental de risco (Indulgente, Negligente ou Autoritário), e estilo parental adequado (Autoritativo). Em seguida, os cuidadores com estilo parental de risco (P7 e P8) foram submetidos ao Protocolo para treino parental. Após esse treino, foram realizadas mais duas sessões de observação direta do procedimento de punção venosa para quimioterapia com a criança, com intervalo de no mínimo uma semana. O cuidador classificado com estilo parental adequado (P9) não participou do treino com o objetivo de ter seus dados comparados com os demais; com esse participante também foram realizadas mais duas sessões de observação durante punção venosa, com o intervalo mínimo de uma semana.

\section{Análise dos dados}

As sessões de observação direta foram realizadas por dois observadores independentes, a partir da gravação em áudio da sequência de comportamentos emitidos pelo acompanhante e pela criança. Esses observadores receberam treinamento prévio com a OSBD, por um período de um mês antes de iniciar a coleta de dados. Esse treinamento foi realizado por meio da observação de 30 sessões de punção venosa com acompanhantes que não seriam incluídos como participantes da pesquisa, mas que autorizaram a realização da observação. $\mathrm{O}$ teste de fidedignidade, entre a pesquisadora e sua auxiliar, resultou em $80 \%$ de concordância.

As sessões gravadas foram transcritas na íntegra e os comportamentos registrados foram analisados por amostragem, segundo as normas da OSBD. Considerando-se que cada sessão de punção venosa ocorria dentro de um determinado período de tempo, fez-se o cálculo da taxa de ocorrência de cada categoria de comportamento, dividindo-se o total de emissões pelo tempo da sessão de observação.

Os comportamentos das crianças foram divididos em duas categorias amplas: 1) Comportamentos Não Concorrentes (CNC), definidos como aqueles que facilitam ou não criam obstáculos à realização do procedimento invasivo; e 2) Comportamentos Concorrentes (CC), definidos como aqueles que, de alguma forma, dificultam, atrasam ou impedem a realização do procedimento invasivo por parte do profissional de saúde (Borges, 1999; Costa Junior, 2001).

Para a análise dos comportamentos observados nas crianças, foram considerados os cinco comportamentos mais frequentes registrados em cada categoria. Desse modo, os CNC foram: Olhar Procedimento (OP), Auxiliar no Procedimento (AP), Explorar o Ambiente (EA), Sinalizar Dor (SD), Desviar o olhar (DO). Enquanto os CC foram: Choramingar
(Cm), Chorar (Ch), Comportar-se de Modo Nervoso (CN), Protestar (Pr) e Gritar (Gr).

Os comportamentos dos cuidadores foram classificados em Monitoria Positiva, Monitoria Negativa e Negligência, a partir de Gomide (2006). Considerou-se como Monitoria Positiva os comportamentos em que os cuidadores demonstrassem ter atenção para com as crianças, incluindo demonstrações de afeto e carinho, conversas que encorajassem atitudes positivas das crianças diante do procedimento invasivo, assim como indicassem conhecimento acerca das atividades e necessidades destas. Nessa categoria foram incluídos: Carinho (Ca), Participar do Procedimento (PP) e Fala Positiva (FP). A Monitoria Negativa se caracterizou pelo excesso de fiscalização do cuidador sobre a criança, com instruções muito repetitivas e realizadas em forma de cobrança e briga, podendo ocorrer ainda ameaças que acabaram não sendo cumpridas pelo cuidador posteriormente. Esses comportamentos foram: Conter $(\mathrm{Ct}) \mathrm{e}$ Fala Negativa (FN). No caso da Negligência, foram incluídos comportamentos que indicassem pouca demonstração de afeto e pouca interação entre as partes. Nesses casos, os cuidadores se afastavam do ambiente onde o procedimento estava sendo realizado com a criança, omitindo auxílio à mesma. Nessa categoria foram incluídos: Afastar-se (Af), Ausentar-se (Au) e Fala Desvinculada (FD).

\section{Resultados}

A maioria dos participantes foi classificada com estilo parental de risco, sendo cinco considerados como Indulgentes (P2, P3, P4, P5, P6), dois como Negligentes (P1, P7) e um como Autoritário (P8). Apenas uma participante apresentou estilo parental Autoritativo (P9).

Considerando-se o Total parcial e o Total geral obtidos em cada uma das categorias de comportamentos, observou-se que Monitoria Positiva (Total geral=184,5) apresentou taxas superiores às outras duas categorias, independentemente da condição a que o participante foi exposto. Esse resultado foi encontrado em oito participantes, exceto em P2 - da Condição Rotina - o qual apresentou maior taxa de comportamentos classificados como Negligência (taxa=16,7).

Comparando-se as três condições quanto às taxas observadas na categoria Monitoria Positiva, os participantes da Condição Treino apresentaram taxas mais elevadas $(67,8)$ do que os participantes da Condição Rotina $(50,3)$, e similares aos da Condição Manual $(66,4)$.

Dentre os participantes da Condição Rotina, P1 apresentou maior taxa de comportamentos negligentes na primeira sessão de observação $(5,6)$, os quais diminuem de ocorrência ao longo das sessões subsequentes $(3,1 ; 2,6 ; 2,1)$. Na segunda sessão, observou-se mesma taxa entre Negligência e Monitoria Positiva $(3,1)$; e, na terceira e na quarta sessões, a taxa de Monitoria Positiva $(5,9)$ foi superior às demais $(0$; 2,1). O participante P2 apresentou em todas as sessões taxas superiores de comportamentos negligentes comparados à Monitoria positiva; entretanto, não apresentou ocorrência de Monitoria Negativa. Por sua vez, P3 apresentou, nas três sessões, taxas maiores de Monitoria Positiva $(5,9 ; 7,1 ; 6,9)$ ao serem comparadas com Monitoria Negativa $(0 ; 0,2 ; 0,4)$ e Negligência $(1,4 ; 0,1 ; 2,0)$. 


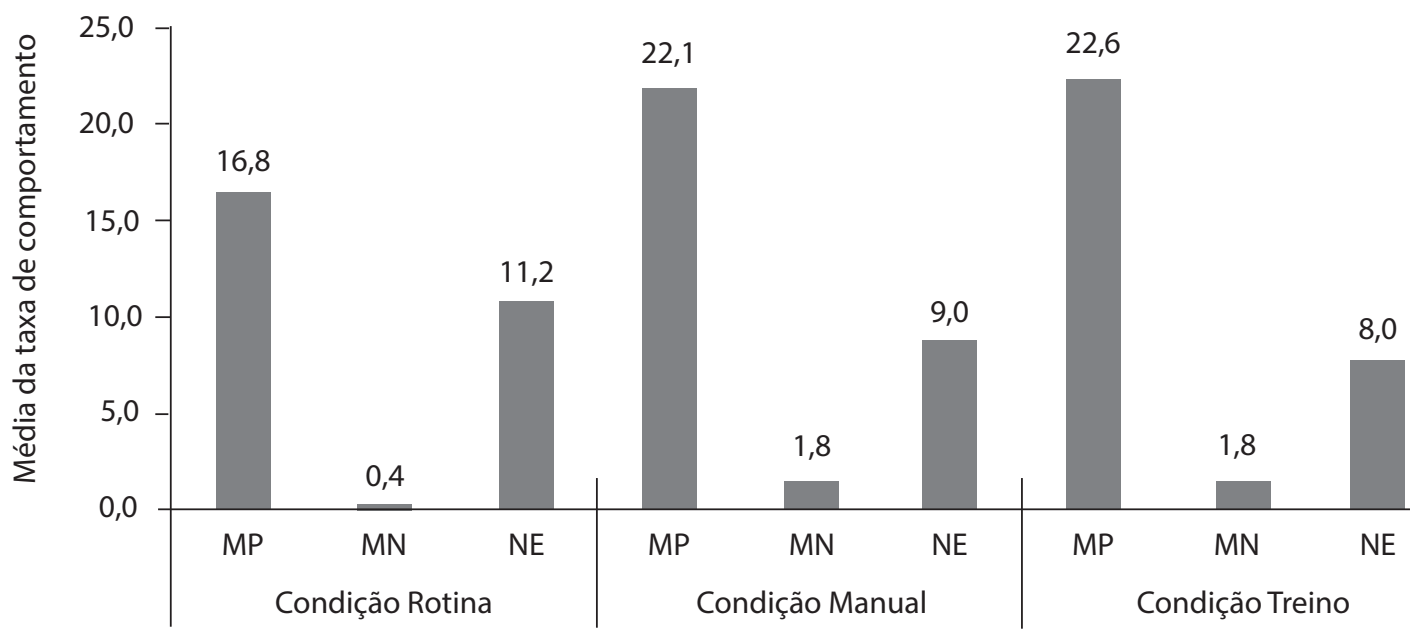

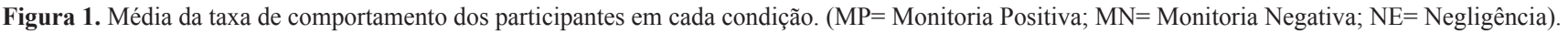

Os três participantes da Condição Manual apresentaram, em cada uma das quatro sessões de observação, taxas de comportamentos de Monitoria Positiva superiores às demais. Observa-se que na segunda sessão, realizada após a apresentação do Manual, houve aumento nas taxas dos comportamentos de Monitoria Positiva nos participantes P5 (de 3,3 para 5,1) e P6 (de 6,8 para 7,6). Entretanto, o mesmo não ocorreu com P4 (cujas taxas reduziram de 7,0 para 6,0). Em relação à Monitoria Negativa, observa-se que $\mathrm{P} 4$ manteve taxas reduzidas ao longo das três sessões posteriores à apresentação do Manual $(0,1 ; 0,2 ; 0,0)$; P5 apresentou aumento de taxa na terceira sessão (de 0,0 para 1,3 ) e P6 somente apresentou taxa de Monitoria Negativa (no valor de 1,3) na terceira sessão de observação. Quanto à Negligência, P4 aumentou a taxa (de 0,0 para 2,7) na segunda sessão (após a introdução do Manual) e manteve valores semelhantes nas demais sessões; P5 apresentou redução de taxa na terceira sessão (de 2,9 para 0,7), mas expressivo aumento (de 0,7 para $8,9)$ na quarta sessão; P6 apresentou diminuição somente na taxa observada na terceira sessão (de 1,3 para 0,6$)$ mantendo taxas semelhantes na primeira, segunda e quarta sessões de observação. Dessa forma, foi possível observar mudanças de comportamento após o Manual apenas em curto prazo, nos participantes P5 e P6.

Quanto aos participantes da Condição Treino, observou-se que P7 apresentou, nas duas primeiras sessões, maiores taxas de Negligência $(5,4$ e 6,3) em comparação à Monitoria Positiva $(3,0$ e 2,2$)$ e à Monitoria Negativa $(0,0$ e 0,0$)$, sugerindo não ter havido modificação em seu comportamento após a aplicação do Manual. Entretanto, na terceira e na quarta sessões, realizadas após o Treino Parental, houve aumento nas taxas de Monitoria Positiva (de 2,2 para 6,2 e 6,6) assim como diminuição nas taxas de Negligência (de 6,3 para 1,0 e 0,9 ). Tais resultados demonstram efeito positivo do treino parental a curto e médio prazo nessa participante.

A participante P8 manteve taxas elevadas de Monitoria Positiva em todas as quatro sessões de observação $(6,1 ; 5,2$; 8,4 e 7,1). Comparando-se os resultados obtidos na primeira e na segunda sessão, após a introdução do Manual, houve diminuição na taxa de Negligência (de 1,9 para 0,2) e aumento na taxa de Monitoria Negativa (de 0,1 para 2,0). Também é possível notar que, na terceira sessão, realizada após o Treino Parental, houve aumento na taxa de Monitoria Positiva (de 5,2 para 8,4), diminuição na taxa de Monitoria Negativa (de 2,0 para 0,0 ) e manutenção na taxa de Negligência (no valor de 0,2$)$. Na quarta e última sessão, a Monitoria Positiva continuou mantendo taxa maior que as observadas na primeira e na segunda sessão $(7,1)$, enquanto Monitoria Negativa apresentou aumento de taxa (de 0,0 para 1,0) e Negligência manteve taxa semelhante à observada na terceira sessão (de $0,2$ para 0,6$)$, não atingindo os valores iniciais.

A participante P9 foi a única avaliada pelo PBI como Autoritativa (isto é, estilo parental adequado), portanto, não foi submetida ao treino parental. Observou-se que essa participante apresentou taxas elevadas de Monitoria Positiva em todas as sessões de observação $(6,1 ; 4,9 ; 6,7$ e 5,3). Após a introdução do Manual, na segunda sessão, ocorreu diminuição na taxa de Monitoria Negativa (de 0,6 para 0,0 ) e aumento na taxa de Negligência (de 1,9 para 3,8). Na terceira e quarta sessões, observou-se aumento em Monitoria Negativa (valor de 1,3 ) e redução em Negligência (valor de 0,9 ).

Na Figura 1 é possível observar a média das taxas de comportamentos apresentadas pelos participantes de cada condição.

Observa-se, na Figura 1, que as médias das taxas da categoria Monitoria Positiva (MP) foram maiores na Condição Manual e na Condição Treino, nas quais foram introduzidas intervenções com os cuidadores das crianças, ao serem comparadas com as médias observadas na Condição Rotina. Quanto às médias das taxas observadas na categoria Negligência (NE), foram mais elevadas na Condição Rotina, em comparação com as demais condições. Por outro lado, as médias das taxas da categoria Monitoria Negativa (MN) foram mais elevadas nas Condições Manual e Treino do que na Condição Rotina.

Considerando-se que as categorias classificadas como Monitoria Positiva (MP) apresentaram taxas mais elevadas em todas as quatro sessões de observação ao serem comparadas com Monitoria Negativa (MN) e Negligência (NE), fez-se uma análise comparando-se as taxas médias obtidas em cada sessão de observação e de acordo com a condição a qual o participante 


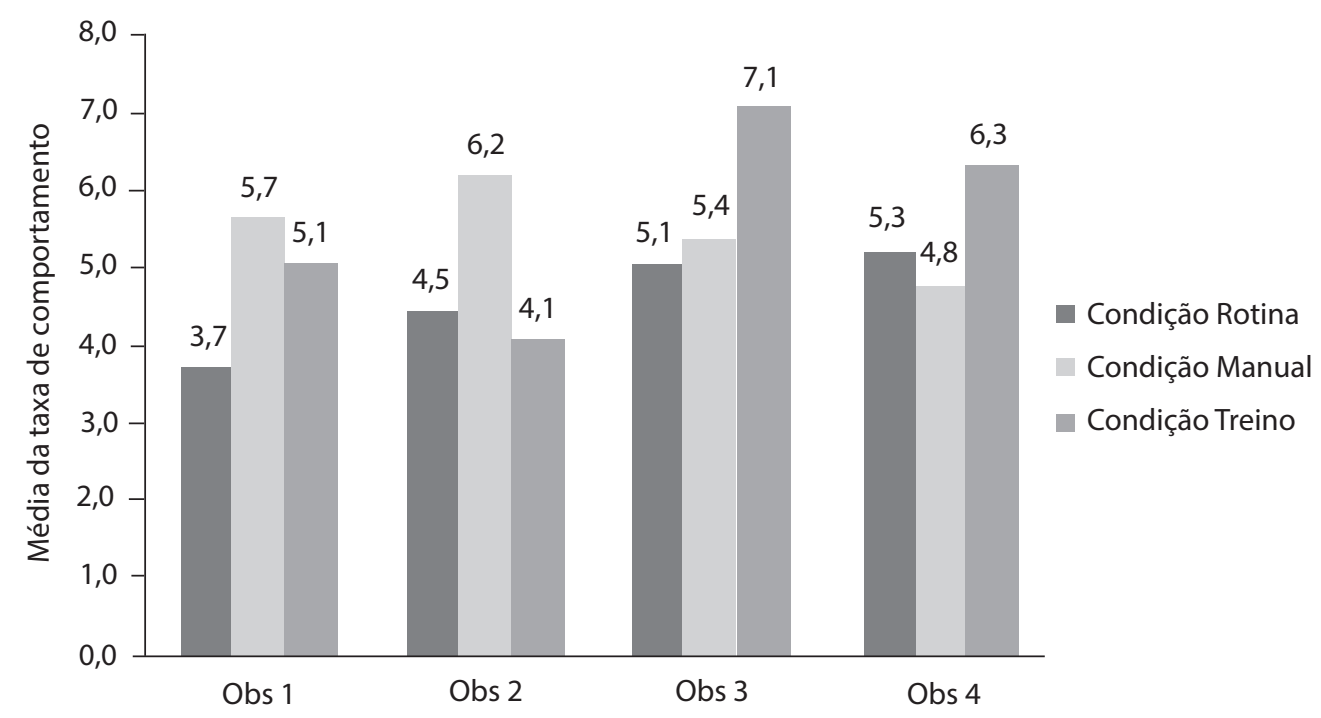

Figura 2. Média da taxa de Monitoria Positiva obtida em cada sessão de observação e de acordo com a condição à qual o participante foi exposto.

foi exposto. Na Figura 2 pode-se observar, mais detalhadamente, a média da taxa da categoria Monitoria Positiva (MP), em cada condição, ao longo das quatro sessões de observação.

Observa-se que na Condição Rotina a categoria Monitoria positiva apresentou um discreto aumento ao longo das sessões (taxas $=3,7 ; 4,5 ; 5,1$ e 5,3), mas apresentando a menor média nas sessões 1 e 3, comparada com as outras duas condições. $\mathrm{Na}$ Condição Manual, os participantes apresentaram a maior média na segunda sessão, logo após a apresentação do Manual; entretanto, este efeito não se manteve nas sessões subsequentes. Os resultados obtidos na Condição Treino demonstram que, na terceira sessão, após a realização do treino parental, esses participantes apresentaram a maior média de MP ao serem comparados com os participantes das outras condições, permanecendo semelhante na quarta sessão.

Fez-se, também, a comparação entre os resultados obtidos com os dois participantes que foram submetidos ao treino parental com os obtidos pelo participante P9 (estilo parental adequado). Na Figura 3 estão dispostas as médias das taxas de Monitoria positiva obtidas com os participantes P7 e P8 e as médias do participante P9 ao longo das quatro sessões de observação.

É possível observar, na Figura 3, que após a realização do treino (terceira sessão), a média da taxa de comportamento de Monitoria positiva aumentou expressivamente, mantendo-se alta tanto na terceira $(7,3)$ quanto na quarta $(6,9)$ sessão de observação.

Na Tabela 1 estão dispostas as taxas dos Comportamentos Concorrentes (CC) e dos Comportamentos Não Concorrentes (CNC) observados em cada criança, nas quatro sessões de observação, assim como o tempo de duração e o número de tentativas realizadas para a punção venosa.

Observa-se que as crianças apresentaram um total geral de taxas de CNC $(148,4)$ maior que o de CC $(67,1)$. Este

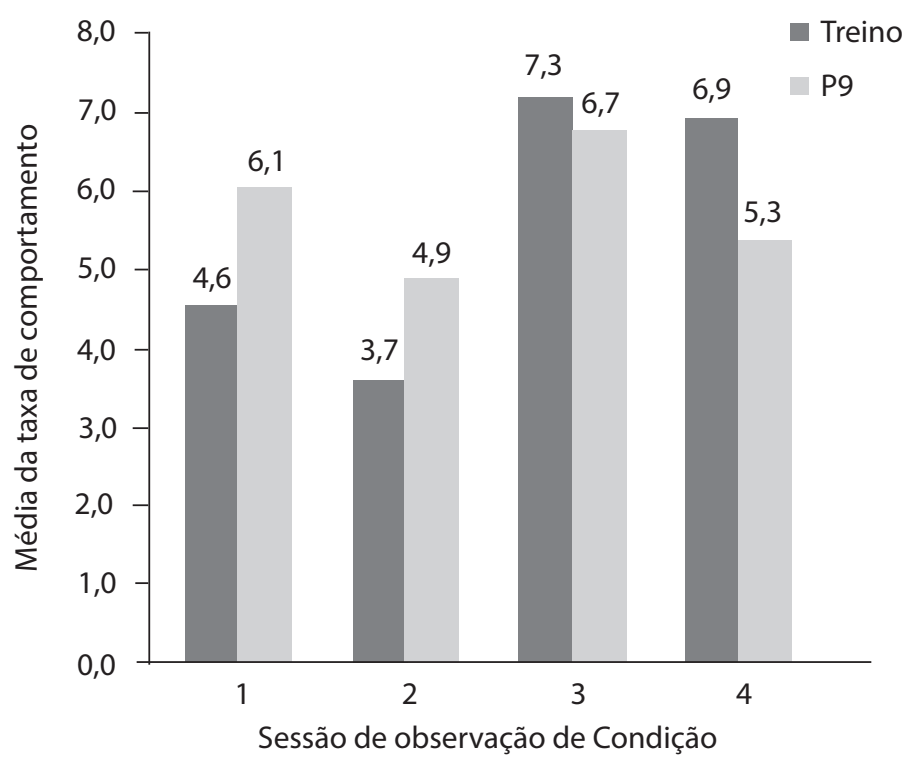

Figura 3. Comparação da taxa de Monitoria Positiva entre os participantes da Condição Treino, em cada sessão de observação. 
Tabela 1.Taxas de comportamentos concorrentes e de comportamentos não concorrentes, tempo de duração de cada sessão de observação e número de tentativas da punção venosa por criança em cada condição

\begin{tabular}{|c|c|c|c|c|c|c|}
\hline Condição & Criança & Observação & $\mathrm{CC}$ & NCC & Tempo(m) & Tentativa \\
\hline \multirow[t]{16}{*}{ Rotina } & \multirow[t]{5}{*}{$\mathrm{C} 1$} & $1^{\mathrm{a}}$ & 2,5 & 3,9 & 3,6 & 1 \\
\hline & & $2^{\mathrm{a}}$ & 2,6 & 5,4 & 3,5 & 1 \\
\hline & & $3^{\mathrm{a}}$ & 3,2 & 4,9 & 4,7 & 1 \\
\hline & & $4^{\mathrm{a}}$ & 0,8 & 7,4 & 2,4 & 1 \\
\hline & & $\Sigma$ & 9,0 & 21,6 & & \\
\hline & \multirow[t]{5}{*}{$\mathrm{C} 2$} & $1^{\mathrm{a}}$ & 0,2 & 4,0 & 4,5 & 1 \\
\hline & & $2^{\mathrm{a}}$ & 0 & 4,2 & 3,5 & 1 \\
\hline & & $3^{\mathrm{a}}$ & 0 & 5,6 & 3,4 & 1 \\
\hline & & $4^{\mathrm{a}}$ & 0 & 2,3 & 2,6 & 1 \\
\hline & & $\Sigma$ & 0,2 & 16,1 & & \\
\hline & \multirow[t]{6}{*}{$\mathrm{C} 3$} & $1^{\mathrm{a}}$ & 3,6 & 3,9 & 2,2 & 1 \\
\hline & & $2^{\mathrm{a}}$ & 4,5 & 1,8 & 17,4 & 2 \\
\hline & & $3^{\mathrm{a}}$ & 2,4 & 6,0 & 4,5 & 1 \\
\hline & & $4^{\mathrm{a}}$ & $*$ & $*$ & $*$ & $*$ \\
\hline & & $\sum$ & 10,6 & 11,7 & & \\
\hline & & Total Parcial & 19,6 & 22,6 & & \\
\hline \multirow[t]{16}{*}{ Manual } & \multirow[t]{5}{*}{$\mathrm{C} 4$} & $1^{\mathrm{a}}$ & 4,0 & 8,0 & 1 & 1 \\
\hline & & $2^{\mathrm{a}}$ & 0,3 & 6,0 & 10 & 2 \\
\hline & & $3^{\mathrm{a}}$ & 0,2 & 6,6 & 5 & 1 \\
\hline & & $4^{\mathrm{a}}$ & 0 & 6,0 & 5,8 & 1 \\
\hline & & $\sum$ & 4,5 & 26,6 & & \\
\hline & \multirow[t]{5}{*}{ C5 } & $1^{\mathrm{a}}$ & 3,0 & 3,4 & 3,7 & 1 \\
\hline & & $2^{\mathrm{a}}$ & 0 & 6,9 & 2,4 & 1 \\
\hline & & $3^{\mathrm{a}}$ & 5,6 & 2,6 & 3 & 1 \\
\hline & & $4^{\mathrm{a}}$ & 4,9 & 4,3 & 15,2 & 3 \\
\hline & & $\Sigma$ & 13,4 & 17,2 & & \\
\hline & \multirow[t]{6}{*}{ C6 } & $1^{\mathrm{a}}$ & 0,5 & 4,9 & 4 & 1 \\
\hline & & $2^{\mathrm{a}}$ & 0 & 5,6 & 7,2 & 1 \\
\hline & & $3^{\mathrm{a}}$ & 4,3 & 1,8 & 4 & 1 \\
\hline & & $4^{\mathrm{a}}$ & 0 & 6,2 & 3.7 & 1 \\
\hline & & $\sum$ & 4,8 & 18,2 & & \\
\hline & & Total Parcial & 22,7 & 62,3 & & \\
\hline
\end{tabular}


Continuação...

\begin{tabular}{|c|c|c|c|c|c|c|}
\hline \multirow[t]{17}{*}{ Treino } & $\mathrm{C} 7$ & $1^{\mathrm{a}}$ & 2,0 & 5,8 & 3 & 1 \\
\hline & & $2^{\mathrm{a}}$ & 0 & 7,7 & 2,7 & 1 \\
\hline & & $3^{\mathrm{a}}$ & 3,5 & 5,3 & 3,7 & 1 \\
\hline & & $4^{\mathrm{a}}$ & 3,9 & 3,3 & 3,3 & 1 \\
\hline & & $\sum$ & 9,5 & 22,1 & & \\
\hline & $\mathrm{C} 8$ & $1^{\mathrm{a}}$ & 3,4 & 4,1 & 6,7 & 1 \\
\hline & & $2^{\mathrm{a}}$ & 3,9 & 3,4 & 5,6 & 2 \\
\hline & & $3^{\mathrm{a}}$ & 2,4 & 7,0 & 4,2 & 1 \\
\hline & & $4^{\mathrm{a}}$ & 4,2 & 2,3 & 5,2 & 1 \\
\hline & & $\sum$ & 14,0 & 16,8 & & \\
\hline & $\mathrm{C} 9$ & $1^{\mathrm{a}}$ & 0,3 & 4,6 & 3,1 & 1 \\
\hline & & $2^{\mathrm{a}}$ & 0 & 6,3 & 3,5 & 1 \\
\hline & & $3^{\mathrm{a}}$ & 0,3 & 7,9 & 3,1 & 1 \\
\hline & & $4^{\mathrm{a}}$ & 0,6 & 5,8 & 3,2 & 1 \\
\hline & & $\sum$ & 1,3 & 24,6 & & \\
\hline & & Total Parcial & 24,8 & 63,5 & & \\
\hline & & Total Geral & 67,1 & 148,4 & & \\
\hline
\end{tabular}

Nota: C: criança; CC: Comportamento Concorrente; NCC: Comportamento Não Concorrente; m: minutos; *: Sessão de observação não realizada em virtude de agravamento no estado clínico da participante.

resultado foi obtido na maioria das sessões de observação (isto é, em 28 de 35 sessões). Nota-se, também, que a duração das sessões foi em média de 4,7 minutos, variando de um até 17,4 minutos. Das 35 sessões de observação, em $88,5 \%(\mathrm{n}=31)$ as punções foram realizadas com apenas uma tentativa.

$\mathrm{Na}$ Condição Rotina, observa-se que as crianças $\mathrm{C} 1$ e C3 apresentaram $\mathrm{CC}$ em todas as sessões, ainda que em categorias e taxas diferentes. Por sua vez, a criança $\mathrm{C} 2$ apresentou apenas um CC ( $\mathrm{PR}=$ Protestar), registrado na primeira sessão de observação, mantendo maior taxa de CNC.

$\mathrm{Na}$ Condição Manual observou-se redução dos CC nas três crianças desta condição na segunda sessão, realizada após a apresentação do Manual com os cuidadores. Nas sessões seguintes, a baixa taxa de CC manteve-se apenas na criança $\mathrm{C} 4$. A criança $\mathrm{C} 5$ apresentou taxas de concorrentes mais altas que as de não concorrentes na terceira e quarta observação. A criança C6 apresentou aumento na taxa de concorrentes na terceira observação e ausência de tais comportamentos na quarta, sugerindo não haver efeito do uso do Manual com os cuidadores sobre o comportamento dessas crianças.

$\mathrm{Na}$ Condição Treino é possível observar que a criança C9, filha da cuidadora de estilo parental autoritativo (adequado) e que não passou pelo treino, apresentou taxas mais altas de CNC em todas as sessões de observação, em comparação com os CC. As crianças $\mathrm{C} 7$ e C8, cujas mães participaram do treino parental, apresentaram resultados diversificados. Após a apresentação do Manual com o cuidador de C7, essa criança apresentou redução dos comportamentos concorrentes (de 2,0 para zero no total); entretanto, após o treino parental com a mãe, $\mathrm{C} 7$ apresentou aumento da taxa de $\mathrm{CC}$, chegando a ser superior à taxa apresentada na primeira observação. A criança C8 não apresentou mudança nas taxas de comportamentos na segunda sessão, após o Manual; entretanto, na terceira sessão, logo após o treino parental, C8 apresentou redução de CC (de 3,9 para 2,4 no total) e aumento dos CNC (de 3,4 para 7,0 no total). Na quarta sessão, houve aumento dos $\mathrm{CC}$ e redução dos $\mathrm{CNC}$, sugerindo não ter havido efeito de manutenção do treino parental em longo prazo.

\section{Discussão}

Este estudo foi realizado com o objetivo de avaliar os efeitos de instrução (Condição Manual) e de treino parental (Condição Treino) sobre comportamentos observados em cuidadores e crianças com diagnóstico de câncer durante procedimento de punção venosa para quimioterapia em ambulatório. Para isso, os resultados foram comparados com os obtidos com participantes submetidos somente à rotina do atendimento hospitalar (Condição Rotina). Também foi investigada a ocorrência de mudanças de comportamentos das crianças como subproduto dos procedimentos de intervenção com os cuidadores.

Os comportamentos mais frequentemente observados nos cuidadores foram os classificados como Monitoria positiva. Nas crianças, foram os classificados como Comportamentos não concorrentes. Tais resultados foram observados 
independentemente da condição à qual os participantes foram expostos.

A princípio, esses resultados podem ser explicados pelas características do contexto em que o estudo foi realizado: punção venosa em ambulatório para tratamento quimioterápico de crianças com câncer acompanhadas pelo cuidador principal. Esse contexto tem sido apontado pela literatura como doloroso tanto para o paciente quanto para o acompanhante, especialmente em se tratando de crianças, as quais tendem a exibir distresse comportamental (Álvarez \& Marcos, 1997; Costa Junior, 1999). Nesses casos, a criança se encontra fragilizada pela doença e exposta à dor decorrente dos procedimentos, em ambiente social como é o caso do ambulatório, sendo esperado que o acompanhante apresentasse predominantemente comportamentos socialmente mais aceitos, como os que foram classificados neste estudo como Monitoria positiva. Por sua vez, o padrão comportamental exibido pelas crianças permitiu que o procedimento fosse realizado. Entretanto, foram observadas diferenças individuais entre os participantes.

Comparando-se as três condições, observou-se que na Condição Manual e na Condição Treino os comportamentos mais observados nos cuidadores foram os classificados como Monitoria positiva seguidos de Monitoria negativa. Os comportamentos classificados como Negligência foram mais observados nos cuidadores da Condição Rotina.

A partir dos resultados obtidos com o Instrumento de Avaliação das Relações Familiares (PBI), a maioria dos cuidadores apresentou estilo parental permissivo (sendo cinco indulgentes e dois negligentes). Esses cuidadores também apresentaram altas taxas de comportamentos de Negligência. Esses resultados coincidem com os de outras pesquisas (e.g. Piccinini et al., 2003; Wakefield et al., 2010) que demonstram um alto grau de permissividade em cuidadores de crianças enfermas.

Em relação aos pais com estilo parental permissivo, a literatura (e.g. Herman \& Miyazaki, 2007) chama atenção para a possibilidade de esses cuidadores prejudicarem a adesão ao tratamento ao se esquivarem da responsabilidade de impor limites a uma criança enferma. Segundo Vance e Eiser (2004), pode ocorrer ainda de esses cuidadores não saberem como reagir diante de comportamentos concorrentes da criança (o que pode explicar a ocorrência do comportamento Afastar-se [AF] observado na cuidadora P7 antes do treino parental).

Nesse sentido, intervenções psicológicas que mostrem alternativas de estratégias de enfrentamento (Christensen \& Fatchet, 2002), ou formas mais eficientes de se comportar diante dos estímulos estressores podem ser extremamente úteis, como, por exemplo, o treino proposto pelo presente estudo.

Neste estudo observou-se que a cuidadora P9 apresentou alta frequência de comportamentos de Monitoria positiva em todas as sessões de observação, assim como a criança C9 apresentou alta frequência de Comportamentos não concorrentes em todas as sessões. Tais resultados apontam que pais com estilo parental autoritativo podem apresentar comportamentos mais adequados frente a situações aversivas, assim como seus filhos, confirmando estudos como o de Sapienza et al. (2009).
Destaca-se que o estilo parental adequado tem sido considerado como uma variável de proteção à criança com câncer, aumentando a probabilidade de emissão de comportamentos colaborativos durante a realização de procedimentos invasivos, seja pela segurança/apoio que o cuidador proporciona nesse momento, seja pela longa história de modelação. Tais resultados poderiam ser investigados em estudos longitudinais que incluíssem pais com estilos autoritativos, comparando-se comportamentos emitidos durante a realização do procedimento invasivo em outros contextos não relacionados a cuidados com a saúde.

Os resultados obtidos na Condição Manual demonstraram efeitos do uso de instrução sobre a aquisição de conhecimentos sobre punção venosa, assim como nas mudanças de comportamentos em curto prazo tanto dos cuidadores quanto das crianças, confirmando estudo de Torres et al. (2009).

Estes resultados diferem dos apontados por Ferreira (2005), no qual foram identificadas mudança e manutenção de comportamentos tanto em curto quanto em longo prazo. Entretanto, ressalta-se que na presente pesquisa foram utilizadas medidas de observação direta do comportamento dos cuidadores e das crianças, enquanto no estudo de Ferreira (2005) as mudanças de comportamento foram obtidas por meio de relatos dos cuidadores após a execução de tarefas e rotinas do tratamento.

$\mathrm{Na}$ Condição Treino, o Manual pareceu não afetar os comportamentos dos cuidadores. Entretanto, após o treino parental, observou-se mudança positiva de comportamentos nas participantes P7 e P8, com aumento e manutenção de Monitoria positiva. Esses resultados foram compatíveis com a literatura encontrada (Rodriguez et al., 2012; Vance \& Eiser, 2004), a qual defende o uso de intervenções focalizadas nos pais (parent coaching) como as mais eficazes e com garantia de mudança de comportamento principalmente se forem individuais e sistematizadas como o treino parental utilizado neste estudo.

Uma das vantagens do treino parental utilizado nesta pesquisa foi a inclusão do treino de habilidades sociais no protocolo, além de técnicas comportamentais dirigidas para o contexto de punção venosa. Observou-se que, as tarefas de casa e o treino de discriminação de comportamentos adequados e de consequenciação positiva proposto no treino, permitiram uma possível generalização destes comportamentos para outros contextos além do hospital, assim como o uso de tais técnicas com os outros filhos, como o que foi relatado pelas cuidadoras $\mathrm{P} 8$ e P9. Esse dado confirma uma das vantagens de utilizar treino com cuidador listadas por Christensen e Fatchet (2002).

A participante $\mathrm{P} 9$, que não foi exposta ao treino parental por ter sido avaliada com estilo autoritativo - adequado segundo a literatura (Peterson et al., 2007; Reichert \& Wagner, 2007; Vance \& Eiser, 2004; Wakefield et al., 2010) - manteve padrão de comportamento predominantemente caracterizado como de Monitora positiva em todas as sessões de observação.

As crianças da Condição Treino apresentaram taxas de comportamentos concorrentes diferenciadas de acordo com o estilo parental de seus cuidadores. Aquelas com cuidadores com estilo de risco (C7 e C8) apresentaram valores mais altos de $\mathrm{CC}$ do que a criança com cuidador com estilo 
parental adequado (C9). Tais resultados sugerem que o treino parental não foi suficiente para promover redução do distresse comportamental das crianças durante a realização do procedimento de punção venosa. Entretanto, sugerem que a história de práticas parentais adequadas (como parece ter sido o caso da díade C9-P9) pode favorecer a modelação do comportamento da criança frente a situações aversivas como é o caso da punção venosa (Rodriguez et al., 2012).

Quanto ao treino parental, algumas dificuldades foram encontradas para a realização do mesmo. Houve mais de 10 recusas em participar do treino com justificativas de não se sentirem à vontade quanto a receber o pesquisador em casa, por residirem no interior ou por difícil acesso ao local de moradia. Acredita-se que, se houvesse um espaço no hospital para realizar os encontros, teria sido possível obter um maior número de participantes.

Outras limitações e dificuldades encontradas na coleta de dados se referiram à mudança de cuidadores da mesma criança ao longo do tratamento, dificuldade de locomoção dos pacientes do interior, a mudança da via do quimioterápico (injeções, comprimidos), interrupção das sessões de quimioterapia por internação, intercorrências ou mesmo quando a criança entrava na fase de manutenção e/ou alta.

Mesmo assim, os resultados obtidos por meio da comparação entre as três condições sugerem a possibilidade de que estudos futuros investiguem a inclusão do protocolo de treino parental na rotina de atendimento de cuidadores de crianças com câncer, observando seus efeitos ao longo do tratamento. Os resultados também sugerem a necessidade de estudos que investiguem os efeitos em longo prazo do uso de manuais de instruções sobre procedimentos invasivos, tanto sobre os comportamentos de cuidadores quanto sobre os comportamentos da criança, pois estudos sistemáticos nessa área ainda são muito escassos.

Conclui-se que o uso de informação auxiliou o cuidador a ter mais conhecimento sobre o procedimento de punção venosa para quimioterapia, além de ter produzido mudança comportamental em curto prazo. O uso do treino parental teve como efeito imediato aumentar a interação dos cuidadores com a criança durante o procedimento, embora não tenha produzido efeito de controle sobre o distresse comportamental da criança. Por outro lado, o treino parental parece ter favorecido um melhor relacionamento entre o cuidador e a criança, generalizando para outros contextos, o que pode ser investigado em futuros estudos.

\section{Referências}

Associação Brasileira de Empresas de Pesquisa [ABEP]. Retrieved from: http://www.abep.org/novo/FileGenerate.ashx?id=252

Álvarez, C. B., \& Marcos, A. F. (1997). Psychological treatment of evoked pain and anxiety by invasive medical procedures in pediatric oncology. Psychology in Spain, 1, 17-36.

Borges, L. M. (1999). Treinamento de respostas de enfrentamento e colaboração em mães de crianças submetidas a procedimentos médicos invasivos. Dissertação de Mestrado. Brasília, DF: Universidade de Brasília.
Brasiliense, I. C. S. (2012). Efeitos do uso de manual de instruções com cuidadores de crianças submetidas à punção venosa para quimioterapia. [Relatório de pesquisa]. Programa Institucional de Bolsas de Iniciação Científica. Belém: Universidade Federal do Pará.

Chen, E., Joseph, M., \& Zeltzer, L. (2000). Behavioral and cognitive interventions in the treatment of pain in children. Pediatric Clinics of North America, 47(3), 513-525.

Christensen, J., \& Fatchet, D. (2002). Promoting parental use of distraction and relaxation in pediatric oncology patients during invasive procedures. Journal of Pediatric Oncology Nursing, 19(4), 127-132.

Costa Junior, A. L. (2001) Análise de comportamentos de crianças expostas à punção venosa para quimioterapia. Tese de doutorado não publicada, Universidade de Brasília, Brasília.

Costa Junior, A. L. (1999). Psico-oncologia e manejo de procedimentos invasivos em oncologia pediátrica: uma revisão de literatura. Psicologia: Reflexão e Crítica, 12(1), 107-118.

Duarte, I. B. (2012). Efeitos de instruções e de treino parental sobre comportamentos observados em cuidadores e em crianças com câncer durante sessão de punção venosa para quimioterapia ambulatorial. Dissertação de mestrado não publicada, Universidade Federal do Pará, Belém.

Ferreira, R. S. (2005). Efeitos da apresentação sistematizada de um manual educativo para pais de crianças com leucemia. Dissertação de mestrado não publicada, Universidade de Brasília, Brasília.

Gomide, P. I. (2006). Inventário de Estilos Parentais (IEP): Modelo teórico, manual de aplicação, apuração e interpretação. Petrópolis: Ed. Vozes.

Herman, A. R., \& Miyazaki, M. C. (2007). Intervenção psicoeducacional em cuidador de criança com câncer: estudo de caso. Arquivo de Ciências da Saúde, 14(4), 238-244.

Jay, S. M., Ozolins, M., \& Elliott, C. H. (1983). Assessment of children's distress during painful medical procedures. Health Psychology, 2(2), 133-147.

Lemos, I. P., \& Ferreira, E. A. (2010). Comportamento de crianças, acompanhantes e auxiliares de enfermagem durante sessão de punção venosa. Psicologia: Teoria e Pesquisa, 26(2), 433-443.

Linhares, M. B., \& Doca, F. N. (2010). Dor em neonatos e crianças: avaliação e intervenções não farmacológicas. Temas em Psicologia, 19(2), 307-325.

Parker, G., Tupling, H., \& Brown, L.B.A. (1979). Parental bolding instrument. British Journal of Medical Psychology. 52, 1-10.

Peterson, A., Cline, R., Foster, T., Penner, L., Parrott, R., Keller, C., ... Albrecht, T. (2007). Parents' interpersonal distance and touch behavior and child pain and distresse during painful pediatric oncology procedures. Journal of Nonverbal Behavior, 31, 79-97.

Piccinini, C.A., Castro, E.K., Alvarenga, P., Vargas, S., \& Oliveira, V.Z. (2003). A doença crônica orgânica na infância e as práticas educativas maternas. Estudo de Psicologia, 8(1), 75-83.

Pinheiro, M., Del Prette, A., \& Haase, V. (2002). Pais como coterapeutas: treinamento em habilidades sociais como recurso adicional. (Relatório Técnico Vol. 3, No 1, pp. 1-42). Belo Horizonte, MG: Universidade Federal de Minas Gerais, Departamento de Psicologia, Laboratório de Neuropsicologia do Desenvolvimento e Laboratório de Psicologia da Família. 
Reichert, C. B., \& Wagner, A. (2007). Autonomia na adolescência e sua relação com os estilos parentais. Psicologia, Saúde \& Doenças, 38(3), 292-299.

Rezende, M.C.L.B. (2001). Estilos parentais e a relação entre pais e filhos com e sem deformidades faciais. Dissertação de mestrado não publicada. Pontifícia Universidade Católica de Campinas.

Rodriguez, E. M., Dunn, M. J., Zuckerman, T., Vannatta, K., Gerhardt, C. A., \& Compas, B.E. (2012). Cancer-related sources of stress for children with cancer and their parents. Journal of Pediatric Psychology, 37(2), 185-197.

Sapienza, G., Aznar-Farias, M., \& Silvares, E.F. (2009). Competência social e práticas educativas parentais em adolescentes com alto e baixo rendimento acadêmico. Psicologia: Reflexão e Crítica, 22(2), 208-213.

Torres, H., Candido, N., Alexandre, L., \& Pereira, F. (2009). O processo de elaboração de cartilhas para orientação do autocuidado no programa educativo em diabetes. Revista Brasileira de Enfermagem, 62(2), 312-6.
Vance, Y., \& Eiser, C. (2004). Caring for a child with cancer - A systematic review. Pediatric Blood Cancer, 42, 249-253.

Wakefield, C. E., McLoone, J., Goodenough, B., Lenthen, K., Cairns, D., \& Cohn, R. (2010). The Psychosocial impact of completing childhood cancer treatment: A systematic review of the Literature. Journal of Pediatric Psychology, 33(3), 262-74.

Weber, L. N., Prado, P. M., Viezzer, A. P., \& Brandenburg, O. J. (2004). Identificação de Estilos Parentais: o ponto de vista dos pais e dos filhos. Psicologia: Reflexão e Crítica, 17(3), 323-331.
Recebido em 28.08.2012

Primeira decisão editorial em 24.05.2013

Versão final em 04.07.2013

Aceito em 18.07.2013 